
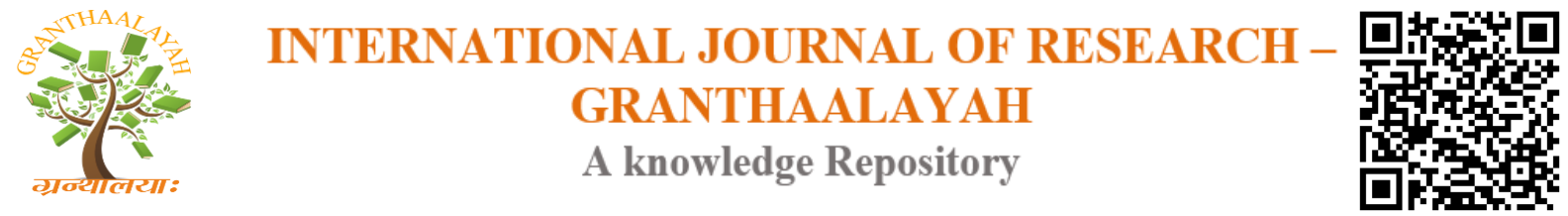

Science

\title{
HIGHLY DISTRIBUTED AND ENERGY EFFICIENT CLUSTERING ALGORITHM FOR WIRELESS SENSOR NETWORKS
}

\author{
Dr. Boselin Prabhu S R ${ }^{* 1}$, Balakumar $\mathbf{N}^{2}$ \\ *1 Assistant Professor, Department of Electronics and Communication Engineering, \\ SVS College of Engineering, Coimbatore, INDIA \\ ${ }^{2}$ Assistant Professor, Department of Electrical and Electronics Engineering, \\ TamilNadu College of Engineering, Coimbatore, INDIA
}

DOI: https://doi.org/10.29121/granthaalayah.v4.i9.2016.2531

\section{ABSTRACT}

Wireless sensor network (WSN) is a low-powered prestigious network fashioned by sensor nodes that treasures application in civilian, military, visual sense models and many others. Reduced energy utilization is an exigent task for these sensor networks. By the data aggregation procedure, needless communication between sensor nodes, cluster head and the base station is eluded. An evaluation of energy efficient optical low energy adaptive clustering hierarchy has been performed and the enactments have been compared with the prevailing low energy adaptive clustering hierarchy algorithm, between two detached wireless sensor network fields. The proposed clustering procedure has been primarily implemented to join two distinct wireless sensor fields. An optical fiber is used to join two reserved wireless sensor fields. This distributed clustering methodology chiefly targets in exploiting the parameters like network lifetime, throughput and energy efficiency of the whole wireless sensor system.

Keywords:

Distributed clustering algorithm, energy efficiency, wireless sensor network, clustering, network lifetime.

Cite This Article: Dr. Boselin Prabhu S R, and Balakumar N, "HIGHLY DISTRIBUTED AND ENERGY EFFICIENT CLUSTERING ALGORITHM FOR WIRELESS SENSOR NETWORKS” International Journal of Research - Granthaalayah, Vol. 4, No. 9 (2016): 30-38.

\section{INTRODUCTION}

Wireless Sensor Networks (WSN) finds applicable in many real-world applications (figure 1) like military, target tracking, environmental monitoring and civilian applications. A WSN can be generally styled as a network of nodes that cooperatively sense and regulates the environment. The activity of sensing, processing and communication under restricted amount of energy projects a need to pattern distributed mechanisms for data processing, MAC and communication protocols. Generally, a wireless sensor node is powered by limited- powered battery. Much of 
the energy consumption takes place during wireless communications. On the other hand, data processing in WSN requires consuming tasks to be accomplished to avoid unnecessary processing power. Energy efficiency can be accomplished at different levels starting from physical layer, MAC layer and routing protocols up to the application level. The concerning protocols in WSNs can be classed into three major categories: routing protocols, sleep/awake scheduling protocols and clustering protocols [1]. One prime method to attain energy efficiency is to efficiently group the sensor nodes into clusters. In order to trim down the data transmission time and energy consumption, the sensor nodes are clustered into a number of small groups called clusters. The grouping of sensor nodes is known as clustering. Each and every cluster has a leader which is branded as cluster-head $(\mathrm{CH})$. A CH is also one of the sensor nodes which are basically affluent in resources. The $\mathrm{CH}$ is selected by the sensor nodes in the respective cluster. The $\mathrm{CH}$ may also be pre-assigned by the user. The main advantages of clustering are that it transmits the aggregated data to the sink or base station (BS), provides scalability for large number of nodes and diminishes the energy consumption.

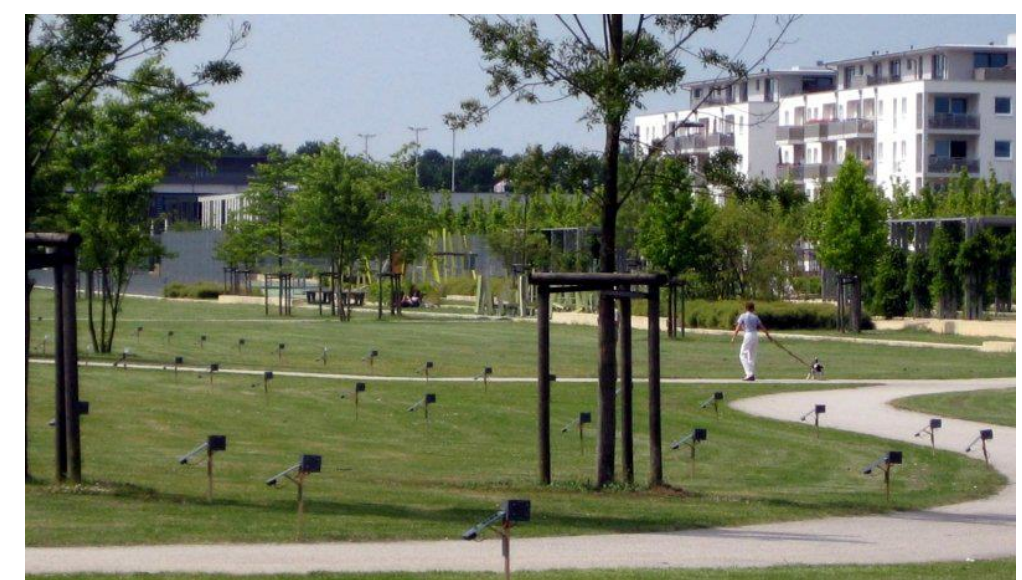

Figure 1: Application of WSN for environmental monitoring.

Clustering may be centralized or distributed, based on the planning of $\mathrm{CH}$. In centralized clustering, the $\mathrm{CH}$ is fixed but in distributed clustering $\mathrm{CH}$ has no fixed architecture. Distributed clustering mechanism is used for some exclusive reasons like sensor nodes prone to failure, better collection of data and minimizing redundant information [2]. Hence these distributed clustering mechanisms have highly self-organizing capability. The rest of this paper has been prearranged as follows. The existing clustering methodologies have been discussed in section II. Section III gives the conceptual description and review of a well-distributed clustering algorithm, the optical low energy adaptive clustering hierarchy $(\mathrm{O}-\mathrm{LEACH})$. Simulation results have been briefed in section IV. Section V concludes the paper and gives few ideas for an improvement over the existing algorithm.

\section{EXISTING CLUSTERING METHODOLOGIES}

Massive research efforts have been taken to lessen the energy consumption and to prolong the lifetime of WSNs. The algorithms described here are utterly distributed and $\mathrm{CH}$ changes from node to node based on some criteria. They diverge in the methodology by which the $\mathrm{CH}$ is elected. Algorithm for Cluster Establishment (ACE) [3] is a highly uniform clustering, selforganizing, effectual coverage, lesser overlapping and emergent cluster forming algorithm for 
WSNs. This is scale-independent and converges in time proportional to the deployment density of the nodes regardless of the overall number of nodes in the network. ACE requires no understanding of geographic location and requires only petite amount of communication overhead [4-6].

In a distributed clustering algorithm, the nodes make autonomous decisions. In Hausdroff Clustering (HC), once cluster formations take place it remains unchanged throughout the network lifetime. Moreover, to evenly use the energy among all the nodes, $\mathrm{CH}$ is rotated among the cluster members. This algorithm expands the lifetime of each cluster in order to increase the life time of the wireless sensor system. $\mathrm{CH}$ selection is essentially based on residual energy of the sensor nodes. Clustering methods have reduced the running down of energy in WSNs. Ringstructured Energy-efficient Clustering Architecture (RECA) [7] uses deterministic CH management pattern to evenly distribute the work load among the nodes within a cluster. Nodes within a cluster make local assessments on the length of their duty cycle according to their remaining energies. RECA is effectual in managing energy in a wide range of networks settings.

Low Energy Adaptive Clustering Hierarchy (LEACH) [8] is a clustering mechanism that distributes energy consumption all along its network; the network is broken down into clusters. The CHs which are purely distributed in manner and the randomly elected CHs, gathers the information from the nodes which are coming under its cluster. It forms clusters based on the received signal strength and uses the $\mathrm{CH}$ nodes as routers to the BS. LEACH also forms one-hop intra and inter cluster topology where each node can transmit directly to the $\mathrm{CH}$ and thereafter to the BS. Consequently, it is irrelevant to networks deployed in wider regions. In the case of TwoLevel Low Energy Adaptive Clustering Hierarchy (TL-LEACH), the CH collects data from other cluster members as original $\mathrm{LEACH}$, but rather than transferring data to the BS directly, it uses one of the $\mathrm{CH}$ s that lie between the $\mathrm{CH}$ and the $\mathrm{BS}$ as a relay station. The primary $\mathrm{CH}$ in each cluster communicates with the secondaries, and the secondaries communicate with the nodes in their sub-cluster. Data fusion is also tasked as in LEACH. The two-level structure of TL-LEACH reduces the number of nodes, which need to transmit data to the $\mathrm{BS}$, effectively lowering the total energy usage.

CLUBS [9] is an algorithm that forms clusters through local broadcast and converge in a time proportional to the local density of nodes. CLUBS can be implemented in asynchronous environment without losing efficiency and simplicity. Furthermore, CLUBS satisfies many constraints that are common in other distributed environment such as limited topology knowledge or access to global IDs. The major problem of CLUBS algorithm is the clusters having their $\mathrm{CHs}$ within 1-hop range of each other. If this is the case, both clusters will collapse and $\mathrm{CH}$ election process will restart. Multi-hop Overlapping Clustering Algorithm (MOCA) [10] is a randomized, distributed multi-hop overlapping clustering algorithm for organizing the sensors into overlapping clusters. The aspiration of the clustering process is to ensure that each node is either a $\mathrm{CH}$ or within the cluster radius from at least one $\mathrm{CH}$. The $\mathrm{CH}$ nomination probability is used to control the number of clusters in the network and the degree of overlap amid them.

In Threshold sensitive Energy Efficient Network (TEEN), at every cluster change time, in addition to the attributes, the $\mathrm{CH}$ broadcasts to its members. This scheme is eminently suited for 
time critical data sensing applications. A smaller value of the threshold gives a more accurate picture of the network, at the expense of improved energy consumption. Thus, the user can govern the trade-off between energy efficiency and accuracy. The decentralized technique, Fast Local Clustering Service (FLOC) [11] is suitable for clustering large-scale wireless sensor networks. It is fast, scalable, produces non-overlapping and approximately equal-sized clusters. This algorithm achieves locality, in that each node is only affected by the nodes within two units. Distributed Weight-based Energy-efficient Hierarchical Clustering (DWEHC) [12] is a well distributed clustering algorithm which spawns well balanced clusters. The high-ranking advantage of DWEHC is that, it shows a vast improvement in both intra-cluster energy consumption and inter-cluster energy consumption.

In O-LEACH algorithm, the infrastructure of a sensor network is composed of a distributed optical fiber sensor (DFS) link and two separated WSN fields. The two WSN fields are crowded with randomly deployed nodes and these nodes can or cannot communicate with each other depending on the required applications. Unlike simple WSNs, since the DFS provide data processing, at one end of the DFS link, the sink or the BS for all WSN nodes is located. The $\mathrm{CH}$ node compress data arriving from nodes that belong to the relevant cluster, and sends the fused data to the BS in order to further reduce the amount of information to be transmitted to the BS. After a given interval of time, to maximize the uniformity of energy consumption of the network, randomized rotation of the role of $\mathrm{CH}$ is conducted. Sensors elect themselves to be local CHs at any time with a certain probability. The O-LEACH algorithm is only a fairly incremental modification to the original LEACH algorithm. Though O-LEACH protocol is comparatively much more energy efficient, the focal drawback in this approach is the random selection of CHs. In the worst case, the $\mathrm{CH}$ nodes may not be evenly distributed among the nodes and it will have its effect on the data gathering. Hybrid Energy Efficient Distributed Clustering (HEED) is a distributed clustering algorithm which selects the $\mathrm{CH}$ based on both residual energy and communication cost. Principally HEED was proposed to avoid the random selection of CHs.

\section{THE OPTICAL LEACH CLUSTERING METHODOLOGY}

In O-LEACH algorithm, the construction of the sensor network encompasses of a distributed fiber sensor link and two detached wireless sensor network fields. Figure 1, displays the disposition of sensor nodes in the two fields. The Distributed Fiber Sensor (DFS) link is positioned at the center of the sensor fields and can cover a certain area. The two WSN fields are occupied with randomly scattered nodes and these nodes can or cannot communicate with each other liable on the required applications. Since the DFS offer data processing, at one end of the DFS link, the base station for all the sensor nodes is situated.

Although the lifetime of the DFS may have to be deliberated as well, the lifetime of such a sensor network is largely determined by the WSN. It consequences that the clustering based algorithm still displays good energy efficiency in the sensor network, particularly when two WSNs are not accessible to each other. As the DFS shelter extended rectangular area, O-LEACH algorithm is used to examine the lifetime of the hybrid sensor network with rectangular topology by isolating and connecting a series of WSNs around the DFS. 
The $\mathrm{CH}$ nodes aggregates the arriving data from the nodes that fit to the suitable cluster and propel the aggregated packet to the base station in order to furthermore trim down the amount of data that must be forwarded to the base station, thereby augmenting the system lifetime. Succeeding to a given interval of time, to adjust the uniformity of energy dissipation of the network, randomized rotation of the concern of cluster head is carried out. Sensors elect themselves to be local cluster heads at any precise time with a definite probability.

As the operation of the standard LEACH algorithm is separated into setup phase and steady phase, O-LEACH operation is likewise separated into two phases, and the steady phase is as same as LEACH. During the setup phase, the selection of cluster head follows the analogous criteria as LEACH, but there are two major alterations between O-LEACH and LEACH: (i) Nodes in WSNs cannot be deployed in the DFS coverage area and (ii) The cluster head and the node must be within the same WSN field if two WSNs cannot communicate with each other.

For most applications, it is superior to assume that the two WSN fields are isolated due to the following reasons: (i) Lengthier data transfer distance over the DFS terrain ends with advanced energy consumption and (ii) Wireless communication over the DFS area is not even permitted for some applications.

Table 1: Simulation Parameter Setup

\begin{tabular}{|l|l|}
\hline Parameter & Values \\
\hline Topology & $500 \times 500 \mathrm{~m}^{2}$ \\
\hline Number of nodes & 30 \\
\hline Data packet size & 4000 bytes \\
\hline Control packet size & 550 bytes \\
\hline Initial energy & 1 Joule \\
\hline Transmitter power & $31.32 \mathrm{~mW}$ \\
\hline Receiver power & $35.28 \mathrm{~mW}$ \\
\hline Ideal power & $712 \mathrm{~mW}$ \\
\hline Sleep power & $144 \mathrm{~mW}$ \\
\hline
\end{tabular}

\section{SIMULATION RESULTS}

The O-LEACH algorithm has been simulated using the network simulator (NS-2). Table 1, displays the necessary simulation parameter setup. In O-LEACH, the base station is expected to be situated at the center of the left edge of the two wireless sensor fields. The network energy dissipation is absolutely a arithmetical behaviour due to the random distribution of WSN nodes. As the DFS is absolutely active, the lifetime of the DFS is not deliberated. Figure 2, demonstrates the network performance in terms of the percentage lifetime of O-LEACH and LEACH.

Initially in 100 rounds, the percentage lifetime is 90 for both the algorithms. In 500 rounds, the percentage lifetimes of O-LEACH and LEACH are 72 and 53 respectively. Similarly in 3000 rounds, the percentage lifetimes of $\mathrm{O}-\mathrm{LEACH}$ and $\mathrm{LEACH}$ are 14 and 1 respectively. The percentage lifetime is found to be linearly increased in $\mathrm{O}-\mathrm{LEACH}$, when compared to LEACH. 
This is because of the usage of DFS link for connecting the sensor fields, which greatly reduces the losses while routing the packets to the base station. It is to be noted that in most practical applications, the DFS coverage is very specific and cannot be freely varied. Sometimes, parallel DFS links may be deployed to cover more broad areas.

Table 2: Percentage lifetime for LEACH and O-LEACH

\begin{tabular}{|c|c|c|c|c|c|c|c|c|}
\hline \multicolumn{2}{|c|}{ Number of Rounds } & 100 & 500 & 1000 & 1500 & 2000 & 2500 & 3000 \\
\hline 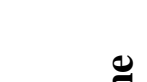 & & 90 & 53 & 40 & 20 & 4 & 2 & 1 \\
\hline 类 & 0 & 90 & 72 & 62 & 43 & 24 & 20 & 14 \\
\hline
\end{tabular}

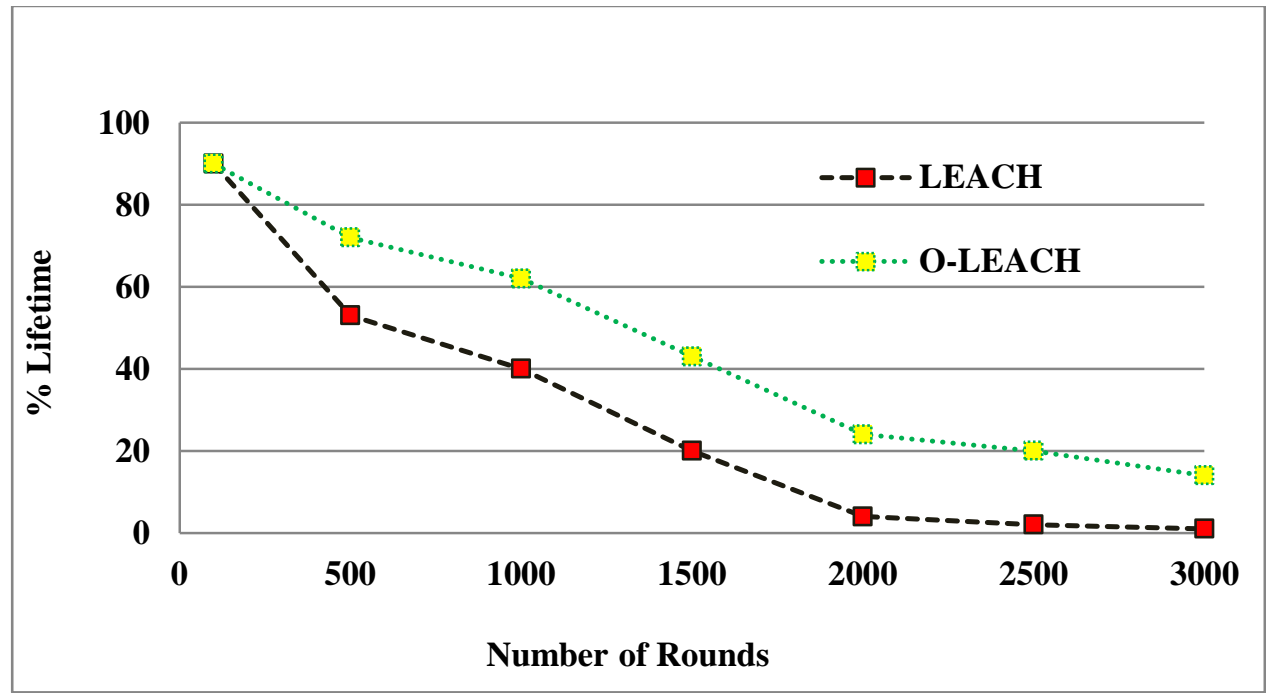

Figure 2: Percentage Lifetime versus Number of Rounds

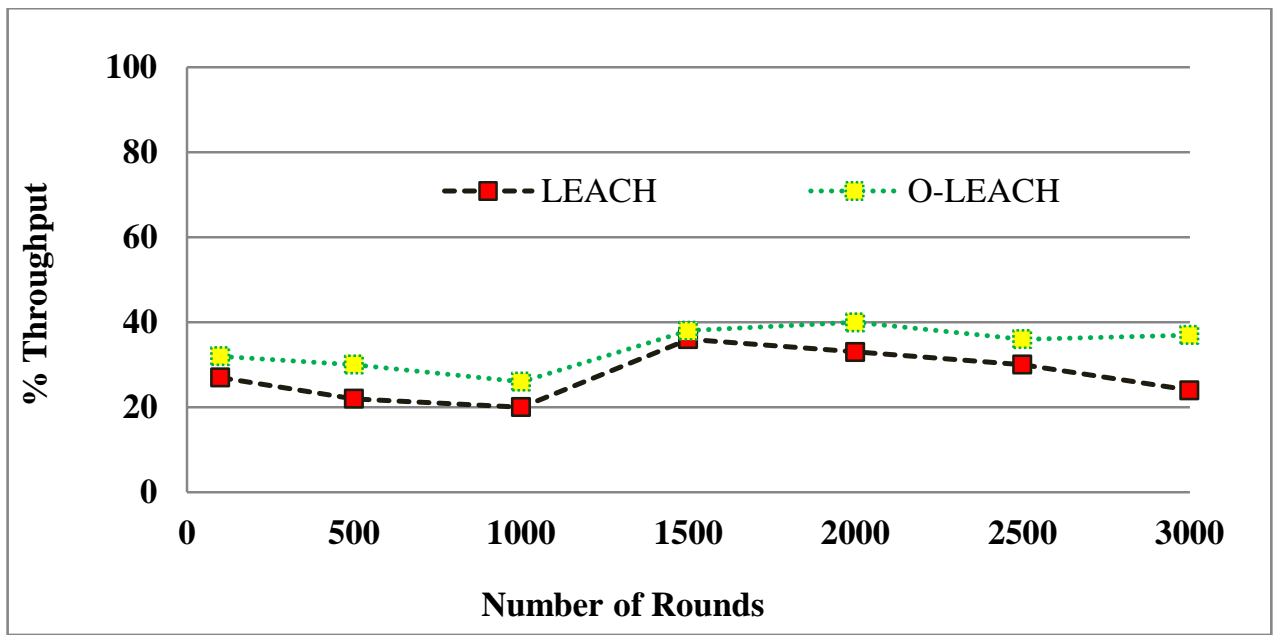

Figure 3: Throughput versus Number of Rounds (O-LEACH and LEACH) 
Table 3: Percentage throughput for LEACH and O-LEACH

\begin{tabular}{|c|c|c|c|c|c|c|c|c|}
\hline \multicolumn{2}{|c|}{ Number of Rounds } & 100 & 500 & 1000 & 1500 & 2000 & 2500 & 3000 \\
\hline \multirow{2}{*}{ 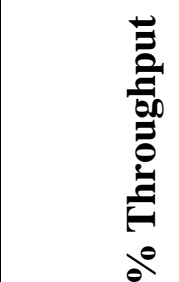 } & 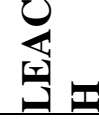 & 27 & 22 & 20 & 36 & 33 & 30 & 24 \\
\hline & ف & 32 & 30 & 26 & 38 & 40 & 36 & 37 \\
\hline
\end{tabular}

Figure 3, shows the performance evaluation of throughput and number of rounds for the two algorithms. Initially in 100 rounds, for O-LEACH and LEACH, the percentage of throughput is 32 and 27 respectively. Similarly in 3000 rounds, the throughput is 37 and 24 for O-LEACH and LEACH respectively. This slight increase in throughput in O-LEACH is mainly due the usage of DFS link for data packet transmission to the base station. Figure 4, shows the energy efficiency comparison of the two algorithms. After 100 rounds, the energy efficiency is found to be improved linearly in O-LEACH when compared to LEACH, for every successive rounds. This clearly indicates that, O-LEACH is efficient mainly when two wireless sensor networks have to be connected together.

Table 4: Energy Efficiency for LEACH and O-LEACH

\begin{tabular}{|c|c|c|c|c|c|c|c|c|}
\hline \multicolumn{2}{|c|}{ Number of Rounds } & 100 & 500 & 1000 & 1500 & 2000 & 2500 & 3000 \\
\hline 힐 & 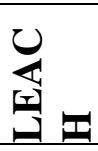 & 42 & 45 & 43 & 46 & 38 & 27 & 20 \\
\hline م & ف & 40 & 35 & 30 & 17 & 12 & 8 & 5 \\
\hline
\end{tabular}

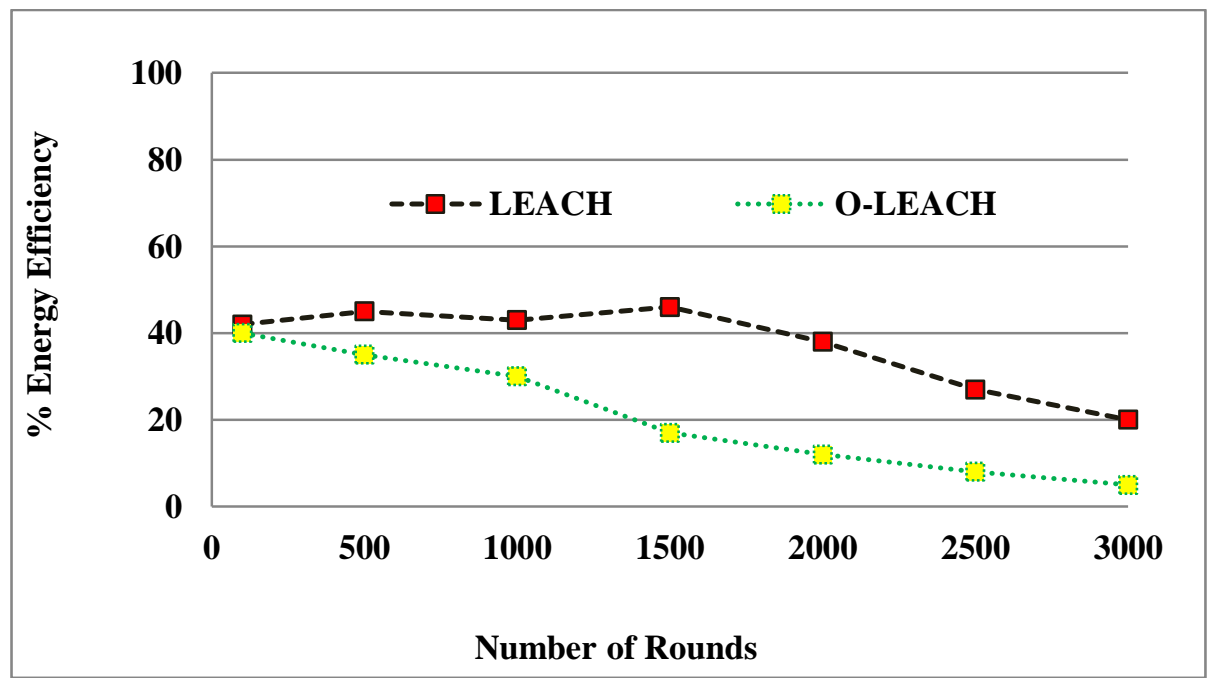

Figure 1: Energy efficiency versus Number of Rounds (O-LEACH and LEACH) 


\section{CONCLUSION}

Generally, a wireless sensor node is powered by limited- powered battery. Much of the energy consumption takes place during wireless communications. On the other hand, data processing in WSN requires consuming tasks to be accomplished to avoid unnecessary processing power. Energy efficiency can be accomplished at different levels starting from physical layer, MAC layer and routing protocols up to the application level. Clustering is the most significant issue for networks where resources are restricted. Thus, it is required to make use of the available energy efficiently. There becomes a need in wireless sensor network to effectively group the sensor nodes in to clusters. In this paper, a review of O-LEACH algorithm has been carried out and the performance evaluation has been done against the existing LEACH algorithm, between two separate wireless sensor network fields. It could be clearly seen that the performance of $\mathrm{O}$ LEACH is comparatively better, thereby O-LEACH is efficient mainly when two separate wireless sensor networks have to be connected together. The optical fiber links leads to various disadvantages like: i) Scattering losses, it is not applicable to use the DFS link for efficient long haul communications, ii) The changes in the light properties usually happen in the sensor, iii) Need for more expensive optical transmitters and receivers, iv) More difficult and expensive to employ splices and v) Difficult to carry electrical power to operate the terminal devices. These drawbacks could be overcome by changing the DFS link with an alternate medium for communication and effectively connecting two separate wireless sensor fields.

\section{REFERENCES}

[1] Akyildiz, I, Su, W, Sankarasubramaniam, $Y \&$ Cayirci, E 2002, 'A survey on sensor networks', IEEE Communications Magazine, vol. 40, no. 8, pp. 102-114.

[2] Ali, M, Voigt, $T$ \& Uzmi, ZA 2006, 'Mobility management in sensor networks', Proceedings of the 2nd International Conference on Distributed Computing in Sensor Systems, pp. 131-140.

[3] Baranidharan, B \& Shanthi, B 2010, 'A survey on energy efficient protocols for wireless sensor networks', International Journal of Computer Applications, vol. 11, no. 10, pp. 0975-8887.

[4] Guo, L, Xie, Y, Yang, C \& Jing, Z 2010, 'Improvement on LEACH by combining adaptive cluster head election and two-hop transmission', Proceedings of the 9th International Conference on Machine Learning and Cybernetics, pp. 11-14.

[5] Haitao, Z, Shiwei, Z \& Wenshao, B 2014, 'A clustering routing protocol for energy balance of wireless sensor network based on simulated annealing and genetic algorithm', International Journal of Hybrid Information Technology, vol. 7, no. 2, pp. 71-82.

[6] Boselin Prabhu, SR \& Sophia, S, 2013, 'Variable power energy efficient clustering for wireless sensor networks', Australian Journal of Basic and Applied Sciences, vol. 7, no. 7, pp. 423-434, ISSN : 1991-8178 (Annexure II) (Serial Number: 2095).

[7] Boselin Prabhu, SR \& Sophia, S, 2012, 'A research on decentralized clustering algorithms for dense wireless sensor networks', International Journal of Computer Applications, vol. 57, no. 20, pp. 35-40, ISSN : 0975- 8887.

[8] Boselin Prabhu, SR \& Sophia, S, 2013, 'Mobility assisted dynamic routing for mobile wireless sensor networks', International Journal of Advanced Information Technology, vol. 3, no. 3, pp. 09-19, ISSN : 2231-1548. 
[9] Boselin Prabhu, SR \& Sophia, S, 2013, 'A review of energy efficient clustering algorithm for connecting wireless sensor network fields', International Journal of Engineering Research and Technology, vol. 2, no. 4, pp. 477-481, ISSN : 2278-0181.

[10] Boselin Prabhu, SR \& Sophia, S, 2013, 'Capacity based clustering model for dense wireless sensor networks', International Journal of Computer Science and Business Informatics, vol. 5, no. 1, pp. 1-10, ISSN : 1694-2108.

[11] Boselin Prabhu, SR \& Sophia, S, 2013, 'Real-world applications of distributed clustering mechanism in dense wireless sensor networks', International Journal of Computing Communications and Networking, vol. 2, no. 4, pp. 99-105, ISSN : 2319-2720.

[12] Han, L 2010, 'LEACH-HPR: An energy efficient routing algorithm for heterogeneous WSN', Proceedings of the IEEE International Conference on Intelligent Computing and Intelligent Systems, pp. 507-511.

\section{AUTHOR BIOGRAPHIES}

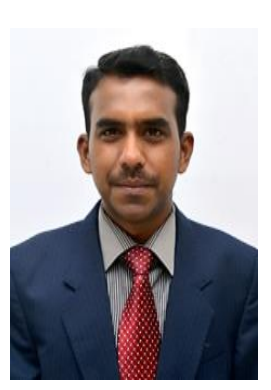

Dr.Boselin Prabhu.S.R obtained his bachelor's degree (B.E) in electronics and communication engineering, master's degree (M.E) in network engineering and doctorate (Ph.D) in wireless sensor networks with the department of information and communication engineering. He is currently working as an Associate Professor with 7 years of experience in teaching and research. His research areas of interest include Wireless Sensor Networks, Mobile Networks and Ad-Hoc Networks. He has published 56 papers in International Journals and Conference Proceedings. He is currently a lifetime member of 46 International Societies. He is an editorial board member, advisory board member and reviewer of 208 International Journals both Scopus and ISI Indexed. $\mathrm{He}$ is the Chief Editor of International Journal of Advanced Engineering and Technology (IJAET). He is an elected fellow member FUAMAE, FISECE, FUAAMP, FISQEM and FUACEE. He has reviewed more than 75 research articles for leading International Journals. He has attained Google scholar citations75 and h-index-05. He is a biographical world record holder of Marquis Who's Who in the World (32 ${ }^{\text {nd }}$ and $33^{\text {rd }}$ Edition) for his outstanding contribution towards research community. He has written one book (electronic circuits-II) for engineering students. He is an Excellent Professional Achievement Award Winner from Society of Professional Engineers in 2016.

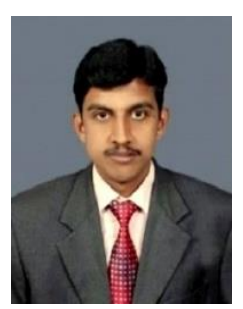

Mr.N.Balakumar earned his Technical degree in Electrical and Electronics Engineering from Sri Ramakrishna Mission Vidhyalaya Polytechnic College. He obtained his bachelor's degree in Electrical and Electronics Engineering and master's degree in Applied Electronics respectively. He is presently working as an Assistant Professor with 5 years of experience in teaching and research. His research areas of interest include Power electronics, Low power VLSI and Embedded system. He has published 09 papers in International Journals and Conference Proceedings. He is currently an active member of PASS, ISOC, IAENG, IRED, CSTA, IAOE, UACSE, ISEIS, IAHF, ITEEA, SDIWC, UAMAE, UAAMP and UACEE. He is an Editorial board member and Reviewer of IJRTER, IJETAE, ISRD, IJECT, IJERT and IRJLTET International Journals. He is elected as a Fellow member of UAMAE (USA). He has reviewed 15 research articles for leading International Journals. 